\title{
Relationship between impulsivity and suicide among the rural elderly in China: A case-control psychological autopsy study
}

\author{
Yunfang Zhou ${ }^{1}$, Zhenyu Ma ${ }^{2}$, Cunxian Jia ${ }^{3}$, Liang Zhou ${ }^{\text {Corresp. } 4}$ \\ ${ }^{1}$ Department of Labor and Social Security, School of Public Administration, Hunan University of Finance and Economics, Changsha, China \\ 2 School of Public Health, Guangxi Medical University, Nanning, China \\ 3 School of Public Health, Shandong University, Jinan, China \\ 4 The Affiliated Brain Hospital of Guangzhou Medical University (Guangzhou Huiai Hospital), Guangzhou, China \\ Corresponding Author: Liang Zhou \\ Email address: Liangzhou_csu@vip.163.com
}

Background : The relationship between impulsivity and suicide is inconsistent in different populations. Hence, the relationship between impulsivity and suicide still needs to be studied among the elderly population. The present study intends to explore the relationship between impulsivity and suicide among the rural Chinese elderly.

Methods: A case-control psychological autopsy study was conducted from February 1, 2014 to December 18, 2015 among rural residents over the age of 60 who died by suicide. The sample consisted of 242 suicides as the case group and 242 living individuals as the control group. Data on demographic characteristics, impulsivity, previous history of suicide attempts, social support, negative life events, and suicidal behavior were collected.

Results: Our study found that impulsivity increased the risk of suicide. The case group showed a higher Barratt Impulsiveness Scale score compared with the control group $(p<0.001)$, which indicates that impulsivity was higher among the elderly suicides. In addition, regression analyses show that impulsivity (odds ratio: 1.03, 95\% confidence interval:1.01-1.06) is an independent risk factor of suicide, after controlling for the effects of marital status, education, family annual income, being left behind, social support, and negative life events. Finally, compared with elderly who do not have a history of attempted suicide, elderly with a history of attempted suicide showed higher impulsivity $(p=0.001)$. 


\section{Relationship between impulsivity and suicide among the rural}

2 elderly in China: A case-control psychological autopsy study

3

4 Yunfang Zhou ${ }^{1}$, Zhenyu $\mathrm{Ma}^{2}$, Cunxian Jia ${ }^{3}$, Liang Zhou ${ }^{4 *}$

$5{ }^{1}$ Department of Labor and Social Security, School of Public Administration, Hunan University

6 of Finance and Economics, Changsha, China

$7 \quad{ }^{2}$ School of Public Health, Guangxi Medical University, Nanning, China

$8{ }^{3}$ School of Public Health, Shandong University, Jinan, China

$9{ }^{4}$ The Affiliated Brain Hospital of Guangzhou Medical University (Guangzhou Huiai Hospital), 10 Guangzhou, China

11

12

13

14

20

21

22

23

24

25

26

27

28

29

30

31

32

33

34

35

36

Corresponding Author: Liang Zhou； 36 Mingxin Road, Liwan District, Guangzhou, China, 510370;

Email address: Liangzhou_csu@vip.163.com 
37 Abstract

38 Background: Conflicting evidence exists on the relationship between impulsivity and suicide,

39 which is rarely studied among the elderly population in rural China. The present study

40 investigated such association in a Chinese rural elderly sample.

41 Methods: A case-control psychological autopsy study was conducted among 242 suicides and

42242 living controls matched for age, gender, and residence. Impulsivity was measured using the

43 Barratt Impulsiveness Scale (BIS). We also collected data on demographic characteristics,

44 previous history of suicide attempts, social support, negative life events, and suicidal behavior.

45 Results: The suicide group had a significantly higher BIS score than the control group

$46(98.79 \pm 16.63$ vs $86.91 \pm 15.18, \mathrm{p}<0.001)$. The higher impulsivity group also had higher rate of

47 past attempted suicide than the lower impulsivity group (25.8\% vs $11.5 \%, \mathrm{p}=0.005)$. Multivariate

48 logistic regression analysis showed that impulsivity was independently associated with suicide

49 with an odds ratio of 1.03 (95\% CI:1.01-1.06), after controlling for all other demographic and

50 psycho-social factors.

51 Conclusions: Our results generally provide support for a positive association between

52 impulsivity and suicide among Chinese rural elderly. The findings provide implications for

53 future intervention programs to identify and treat impulsivity as an effective means for suicide

54 prevention.

55

56

57

58

59

60

61

62 


\section{Introduction}

64 Suicide, which is the act of intentionally taking one's own life (Turecki et al. 2019), has been

65 considered as a major global health problem (Dong et al. 2015). Suicide causes 800, 000 deaths

66 every year and has been listed as the 15th leading cause of death all over the world (WHO 2019).

67 Although the global prevalence of suicide has remarkably dropped in recent years, suicide in

68 China contributes to the overall burden of suicide, accounting for approximately $1 / 5$ of global

69 suicides (Dong et al. 2015). In China, the risk of suicide increases with age, with elderly aged

70 over 65 having the highest suicide rate of 44.3-200 per 100,000, which is 4-5 times higher than

71 the general population (Li et al. 2009). Despite the decreasing suicide rate among the general

72 population, suicide among the elderly population has shown an increasing trend (Sha et al. 2017;

73 Wang et al. 2014). Moreover, the suicide rates of elderly in rural areas are higher than those in

74 urban areas in China (Li \& Katikireddi 2019). China has a rapidly ageing population, but the

75 mental health care system in rural China is still in its infancy. In addition, most rural areas of

76 China are experiencing high work migration where young people move to big cities to look for

77 job opportunities and leave their old parents living in countryside alone. Rural elderly, especially

78 those living alone, are faced with tremendous difficulties and challenges physically, mentally and

79 socially, which put them at higher risk of suicide. Consequently, it is both important and urgent

80 to understand contributing factors that lead to suicide, which is rarely studied among rural

81 elderly population in China.

82 Among a range of risk factors that have been identified to predict suicide, impulsivity is one

83 of the most-commonly mentioned, yet also most controversial factor reported in the literature.

84 There is no standard definition of impulsivity, which has been defined and operationalized in

85 various ways by various researchers (Bakhshani 2014). Moeller et al.(Moeller et al. 2001) looked

86 at impulsivity from a bio-psycho-social perspective and emphasized three essential aspects of 
87 impulsivity: 1) decreased sensitivity to negative consequences of behavior; 2) immediate and 88 unplanned reaction to stimuli before processing the information thoroughly; and 3) no regard for 89 long-term consequences of a behavior. This definition provides helpful insight in guiding for 90 research and treatment on impulsivity and its related risk behaviors.

91 Although impulsivity has been widely recognized as a predisposition towards risky

92 behaviors such as suicide (Bakhshani 2014), studies on the relationship between impulsivity and 93 suicide have yielded inconsistent and variable results. A large number of research have adopted 94 impulsivity as a significant risk factor or waning sigh for suicide. For instance, a literature 95 review by Gvion and Apter (Gvion \& Apter 2011) showed that impulsivity was highly correlated with suicidal behavior across both psychiatric and non-psychiatric populations. Another

97 literature review and meta-analysis on impulsivity in the self-harm and suicidal behavior of young people also demonstrated significant positive associations between multiple facets of impulsivity with suicide behaviors (McHugh et al. 2019). As a result, impulsivity has been highlighted as an important suicide risk factor by many organizations including the American Association of Suicidology, American Foundation for Suicide Prevention and the Substance Abuse and Mental Health Services Administration. However, an almost equally large number of studies have also shown small or even no association between impulsivity and suicide. For example, Smith et al. (Smith et al. 2008) posited that most suicide is not a result of impulsive decisions but involve a plan. This proposition was also endorsed by Anestis et al. (Anestis et al. 2014) who conducted a literature and meta-analysis of the association between trait impulsivity and suicidal behavior and found very small correlations. A recent literature review also found only limited between-group differences in various aspects of impulsivity between suicide and non-suicide group (Beach et al. 2021). Furthermore, Klonsky and May found no association 
110 between impulsivity and suicide in both their own studies and by literature review (Klonsky 111 2015).

112 The inconsistent results on impulsivity and suicide may be partly explained by differences in

113 sample, suicide outcomes (ideation, attempt, and death), measurement of impulsivity, and study

114 design across various studies. However, there have been no studies that examined the association

115 between impulsivity and suicide among older adults in China, who are most susceptible and

116 vulnerable to suicide. A better understanding of suicide and its association with impulsivity

117 among Chinese rural elderly not only contributes more evidence to the already conflicting

118 literature, but also help guide for further intervention programs for the treatment of impulsivity

119 and prevention of suicide. The current study was conducted to fill in the research void by

120 investigating the relationship between suicide and impulsivity among Chinese rural older adults.

121

122 Methods

123 Design and ethical statement

124 The study, conducted from February 1, 2014 to December 18, 2015, utilized a matched case-

125 control study design combined with psychological autopsy method. The study was approved by

126 the Institutional Review Board of the Central South University (Ethical Application Ref: CTXY-

127 130041-1), Shandong University (Ethical Application Ref: 20150306-1), and Guangxi Medical

128 University (Ethical Application Ref: 20150146).

129 Sample and sampling

130 Data on rural residents over the age of 60 who died by suicide were collected in this study.

131 Information on suicide deaths among the elderly, which was used as the case group, was

132 obtained from the local governments' death certification system. For the control group, living 
133 comparisons were matched 1:1 with the suicides in the case group in terms of age ( \pm 3 years),

134 gender, and residence. When a suicide from the case group is identified, we first listed and

135 enumerated all the elderly adults from the same village with matching age and gender. Then, a

136 computer software was used to randomly select one living comparison from the list of matching

137 individuals. In rare instances when no appropriate living comparisons were available, we

138 expanded our search to the nearest villages.

139 A multistage stratified cluster sampling method was adopted to recruit participants. First,

140 Shandong, Hunan, and Guangxi provinces were selected to correspond to eastern, central, and

141 western China, respectively. Second, 12 counties were randomly chosen from the three

142 provinces, including Zoucheng, Junan, and Gaotang from Shandong Province; Yongding, Cili,

143 and Sangzhi from Hunan Province; and Hengxian, Wuming, Lingchuan, Hepu, Luzhai, and

144 Lingyun from Guangxi Province. Elderly residents were recruited, and information on elderly

145 suicides was obtained from each county. Finally, 242 suicides and 242 living individuals were

146 included in this study.

\section{Procedure of the interview}

148 Face-to-face interviews were conducted with two informants of both suicide group and living

149 controls. The two informants usually included one next-to-kin who lived with the subjects, and

150 one non-kin person such as friend, neighbor, or a remote relative. Interviewers from the three

151 research sites received uniform and standard training on psychological autopsy and interview

152 methods before the start of the interviews. Each interview took about 90 minutes with one

153 interviewer interviewing one informant each time. The aims and procedures of the research were

154 explained, and a written informed consent was obtained from the participants at the start of the 
155 interview. Each interview lasted approximately 90 minutes. The details of the participant

156 selection and interview procedures were described in previous publications(Zhou et al. 2019).

157 Measurements

158 Demographic characteristics

159 The demographic data included gender, age, educational level, marital status, family income,

160 physical diseases, and being left behind. Marital status was classified into currently married

161 (including married, remarried and cohabiting) and not currently married (including single,

162 separated, divorced, and widowed). Being left behind is defined as desertion by adult children

16312 months prior to suicide (for the case group) or investigation (for the control group), residence

164 away from the original township for at least 10 months, and infrequent visits (no more than

165 twice).

166 Barratt Impulsiveness Scale

167 Impulsivity was measured using the Barratt Impulsiveness Scale (BIS). It is a 30-item scale

168 designed to assess the personality and behavioral construct of impulsiveness and includes three

169 subscales: non-planning, motor, and attentional impulsivity (Patton et al. 1995). Each item is

170 rated on a five-point Likert scoring from 1 "never" to 5 "very frequently". The total score ranges

171 from 30 to 150 , with a higher score indicating high impulsivity. The original BIS showed good

172 internal consistency with Cronbach's alpha of $0.77-0.89$ and a test-retest reliability of $0.68-$

1730.89 (Patton et al. 1995). The Chinese version of BIS has been used in a previous psychological

174 autopsy study in China and showed satisfactory reliability and validity (Lu et al. 2012).

175 Life Events Scale for the Elderly (LESE)

176 Life events were measured using the Life Events Scale for the Elderly (LESES). It is 46-item

177 scale specifically developed for elderly adults to assess stressful life events during the last 
17812 months before suicide or investigation and includes three subscales: health-related events,

179 family problems, and social communication problems. Each event was recorded from five

180 dimensions: the date it happened, whether it was positive or negative, the impact to mental health

181 of the target person, the duration of the event, and the number of times it happened. The

182 intensity of each life event is calculated by the impact multiplied by the duration and then by the

183 number of times it happened. The total score of negative life events is the sum of all the scores of 184 negative life events, with higher score indicating more negative life events. The Chinese version 185 of LESE has been used in a previous psychological autopsy study in China and showed 186 satisfactory reliability and validity (Mo et al. 2019).

187 Duke Social Support Index

188 Social support was measured using the Duke Social Support Index (DSSI). It is a 23-item scale 189 designed to assess multiple dimensions of social support in the last week before 190 death/investigation. The total score ranges from 11 to 45 , with a higher score indicating higher 191 social support. The Chinese version of DSSI has been used in a previous psychological autopsy 192 study in China and showed satisfactory reliability and validity (Pan et al. 2020).

\section{Suicide Intent Scale}

194 The Suicide Intent Scale (SIS) was used to measure factual aspects of the suicide sttempt, which 195 included the attempters' precautions, planning, communication, and expectations about the 196 suicide behavior (Beck \& Lester 1976). The Chinese version of SIS has been used in a previous 197 psychological autopsy study in China and showed satisfactory reliability and validity (Ma et al. 198 2020; Zhang \& Jia 2007).

199 Features of suicide behavior and history of suicide attempts 
200 Information on the time, place, and method of suicide in the case group were collected. The data 201 obtained included the number of suicide attempts and the timeframe and method of the most 202 recent suicide attempt.

\section{Statistical analysis}

204 The data were analyzed by using SPSS 19.0 software package. Comparisons of parametric data 205 for the two groups were performed using one-way blocked analysis of variance and chi-squared 206 test. The Wilcoxon signed-rank test was used for nonparametric data in the two groups.

207 Conditioned multivariable logistic regression was used to determine the risk factors of 208 suicide. Conditional logistic regression is a specialized type of logistic regression used when case 209 subjects with a particular condition are each matched with $\mathrm{n}$ control subjects without the 210 condition and has become a standard for matched case-control data. In this study, we adopted a 211 1:1 matched case-control study design matching on age, gender and residence. In this 212 conditioned multivariable logistic regression, the dependent variable was suicide (case $=1$, 213 control=0), and the independent variable was impulsivity, while controlling for the following 214 covariates: marital status, being left behind, social support, education, family annual income, and 215 total stimulation of negative life. Physical diseases were not included in the regression because 216 the total stimulation of negative life had included the effect of physical diseases. The adjusted 217 OR and a 95\% CI were used to assess the association between the risk factors and suicide. 218 Finally, $\mathrm{p}<0.05$ was considered statistically significant.

\section{Results}

221 Comparison of socio-demographic and psycho-social characteristics between suicides and 222 living controls 
223

224

225

226

227

228

229

230

231

232

233

234

235

236

237

238

239

240

241

242

243

244

Table 1 shows a comparison of socio-demographic and psycho-social characteristics between 242 pair of matched suicides and living controls. Compared to the control group, the suicide group were more likely to be not currently married (49.6\% vs. $29.8 \%, p<0.001)$, unemployed ( $80.6 \%$ vs. $69.8 \%, p=0.021)$, being left behind $(16.9 \%$ vs. $10.3 \%, p=0.034)$, and having physical diseases $(83.5 \%$ vs. $66.5 \%, p<0.001)$. The suicide group had higher score in negative life events $(49.76 \pm 30.19$ vs $25.24 \pm 28.46, p<0.001)$ and lower score in social support $(22.88 \pm 5.98$ vs $27.47 \pm 6.81, p<0.001)$ than living controls. The suicide group also showed higher scores in overall BIS scores $(98.79 \pm 16.63$ vs. $86.91 \pm 15.18, \mathrm{p}<0.001)$, as well as its three sub-scales (all $\mathrm{p}$ values $<0.001)$.

\section{Comparison of suicidal behaviors between high and low impulsive suicides}

Suicide cases were dichotomized into two groups, higher impulsivity and lower impulsivity group, based on the median of BIS scores. Characteristics of suicide behavior in these two groups were compared (table 2). The proportion of previous suicide attempt was significantly higher in the higher impulsivity group than that in the lower impulsivity group $(25.8 \%$ vs. $11.5 \%, \mathrm{p}=0.005)$, while no significant differences were found in time, location, or means of suicide, or suicide intent between the high and low impulsivity group.

\section{Multivariate logistic regression on risk factors of suicide}

Multivariate logistic regression was used to determine the risk factor of suicide (table 3). Independent variables included in the regression were marital status, being left-behind, income, years of school education, impulsivity, social support, and life events. Four variables entered the final model: impulsivity (OR: 1.03, 95\% CI: 1.01-1.06), marital status (OR: 2.26, 95\% CI: 1.044.92), social support (OR: 0.93, 95\% CI: 0.88-0.99), and life events (OR: 1.02, 95\% CI: $1.01-$ 1.03). We also run additional regressions with each subscales of impulsivity as independent 
246 variable and found only motor impulsivity was independently associated with suicide in the final

247 model (OR:1.08, 95\% CI: 1.03-1.13).

248

249 Discussion

250 In the present study, we found that the suicide group had significantly higher scores in the total

251 and three sub-scale scores of impulsivity than the control group. Within the suicide group, those

252 with higher impulsivity were more likely to have a history of previous suicide attempt than those

253 with lower impulsivity. Conditioned multivariable logistic regression showed that impulsivity

254 was independently associated with suicide, after controlling for socio-demographic

255 characteristics, social support, and life events.

256 Our major finding was that impulsivity was an independent risk factor for suicide among

257 rural older adults in China. This finding was consistent with most studies conducted among

258 youth in both urban and rural areas, and in China and abroad (Florez et al. 2019; Swahn et al.

259 2012; Zhang et al. 2011). For instance, Zhang et al. (Zhang et al. 2011) investigated suicide

260 among rural youth from three provinces using case-control psychological autopsy method and

261 found dysfunctional impulsivity as a risk factor for suicide. When compared with other studies

262 conducted among rural elderly, our finding was in line with some studies while contrasted with

263 others. For instance, Neufeld and O'Rourke (Neufeld \& O'Rourke 2009) examined impulsivity

264 and hopelessness in predicting suicide-related ideation among older adults and found impulsivity

265 played a stronger impact than hopelessness. However, in Liu et al.'s (Liu et al. 2018) research on

266 comparing suicide risk factors between elderly suicides and non-elderly suicides, they found

267 impulsivity as a risk factor for suicide in non-elderly, but not the elderly. While it has been

268 widely recognized that impulsivity is a common feature of youth suicide, impulsivity in elderly

269 suicide is more controversial and needs further research attention to understand the underlying

Peer] reviewing PDF | (2020:07:51178:1:1:NEW 21 May 2021) 
270 mechanism (Demircin et al. 2011). In general, our study finding indicates future development of

271 suicide prevention programs among rural elderly need to pay attention to their impulsivity level,

272 and provide timely assessment and effective treatment to reduce their risk of suicide.

273 This study compared suicidal behavior feature between higher impulsivity and lower

274 impulsivity group. Impulsivity is associated with the history of attempted suicide, which is

275 consistent with the finding of previous studies. Studies have showed that impulsive people are

276 likely to attempt suicide repeatedly(Mann et al. 1999) and that people with repeated suicide

277 attempts are more impulsive than those with first-time suicide attempts (Dougherty et al. 2004).

278 A psychological autopsy study in youth suicides in China shows that the more impulsive

279 population is prone to commit suicide through pesticide (Zhang \& Li 2013). This finding

280 suggests past history of suicide as a risk factor for impulsivity, which may increase future risk of

281 suicide. In future suicide prevention programs, more caution needs to be paid to individuals who

282 had a past history of suicide attempt, with necessary screening of impulsivity and suicide risk

283 conducted to these individuals and more psycho-social support provided to them. However, we

284 do not find significant difference between the suicide means used by suicides with high and low

285 impulsivity, with pesticides suicide being the most commonly means for both groups. The

286 patterns of suicide methods in our study were in keeping with that in Liu et al's study on both

287 elderly and non-elderly suicide (Liu et al. 2018). This finding suggests that easy access to

288 pesticides in rural areas may be a potential facilitator for elderly suicide, regardless their

289 impulsivity level. One implication may be that controlling suicide means such as limiting the

290 purchase of lethal pesticides may serve as an effective preventive strategy for suicide.

291 Aside from impulsivity, our study also demonstrated other important risk factors related to

292 suicide, such as low social support, not being currently married status, and experiencing more 
293 negative life events. This finding was in accordance with most of the previous studies showing

294 poorer living conditions of the rural elderly such as living alone, lower social support and

295 negative life events may aggravate their risk of suicide (Liu et al. 2018). The elderly, especially

296 those who are not married, usually have few social network and receive little social support,

297 which makes them more vulnerable to negative life events and more easily to take extreme risk

298 behaviors such as suicide. It turns out that an abnormal psychological status and the instability of

299 emotion and actions are responsible for the tendency to extreme behavior including suicide. All

300 these findings indicate that suicide is not only the consequence of impulse ridden personality, but

301 also be affected by complicated interaction of various risk factors (Paris 2005). Implications of

302 this finding include more social support, more psycho-education need to be provided to rural

303 elderly to improve their psychosocial well-being, especially among those who had experienced

304 significant negative life events.

305 In interpreting results of the present study, two major limitations should be noted. First, 306 psychological autopsy is a retrospective method that relies on interviews with third-party

307 informants, which has certain shortcomings such as sampling bias, recall bias, external

308 confounders, and lack of standard procedure, and thus may lead to inaccurate assessment and 309 unreliable conclusions (Pouliot \& De Leo 2006). However, psychological autopsy has been in 310 use for over 30 years as a prime approach in identifying risk factors for suicide and is the best 311 available method so far. Besides, we used a case-control study design to collect the data from

312 both the case and control groups using the same method, which may minimize the risks of

313 external confounders. Future research may benefit from combining psychological autopsy with

314 qualitative approaches and interviewing as many proxies of the suicides as possible to further

315 strengthen its reliability and validity (Hjelmeland et al. 2012). Second, in examining risk factors 
316 of suicide, we didn't include some other well-known factors such as mental illness and prior

317 suicide attempt. Although mental illness, especially mood disorder, has been identified as a most

318 prominent predictor for suicide in western world, studies in Asian countries such as China

319 showed different patterns where suicides without a diagnosis of mental illness were more

320 common (Milner et al. 2013). Future research that includes assessment on mental illness

321 diagnosis in Chinese rural elderly suicide population and comparison with other countries is

322 warranted to test such a difference. For previous suicide attempt, we found only a few cases of

323 previous suicide attempt in living controls, which may result in low power of hypothesis testing

324 if included in the multivariate analysis. Future research with larger sample size is needed to

325 examine the association between precious suicide attempt and suicide death with more power.

326

327 Conclusions

328 In conclusion, our finding showed that impulsivity was a significant independent risk factor of suicide 329 among rural elderly in China, which warrants future research to further test such association and examine 330 its underlying mechanism. Future suicide intervention programs may consider adding impulsivity 331 assessment into its routine risk evaluation and treat high risk population in time and properly. In addition, 332 social support may be provided to the elderly, especially those who experienced significant negative life 333 events, to improve their psycho-social well-being and prevent suicide.

334

335

336

337

338

339

340

341

Peer] reviewing PDF | (2020:07:51178:1:1:NEW 21 May 2021) 


\section{References}

346

347

348

349

350

351

352

353

354

355

356

357

358

359

360

361

362

363

364

365

366

367

368

369

370

371

372

373

374

375

376

377

378

379

380

381

382

383

384

385

386

387

388

389

390

391

392
Anestis MD, Soberay KA, Gutierrez PM, Hernandez TD, and Joiner TE. 2014. Reconsidering the link between impulsivity and suicidal behavior. Pers Soc Psychol Rev 18:366-386.

Bakhshani NM. 2014. Impulsivity: a predisposition toward risky behaviors. Int J High Risk Behav Addict 3:e20428.

Beach VL, Gissandaner TD, and Schmidt AT. 2021. The UPPS Model of Impulsivity and Suicide: A Systematic Literature Review. Arch Suicide Res:1-22.

Beck AT, and Lester D. 1976. Components of suicidal intent in completed and attempted suicides. J Psychol 92:3538.

Demircin S, Akkoyun M, Yilmaz R, and Gokdogan MR. 2011. Suicide of elderly persons: towards a framework for prevention. Geriatr Gerontol Int 11:107-113.

Dong $X$, Chang ES, Zeng $P$, and Simon MA. 2015. Suicide in the global chinese aging population: a review of risk and protective factors, consequences, and interventions. Aging Dis 6:121-130.

Dougherty DM, Mathias CW, Marsh DM, Papageorgiou TD, Swann AC, and Moeller FG. 2004. Laboratory measured behavioral impulsivity relates to suicide attempt history. Suicide Life Threat Behav 34:374-385.

Florez IA, Au J, Morrisette N, and Lamis DA. 2019. Risk factors for suicide attempts among rural youth with a history of suicidal ideation. Death Stud:1-7.

Gvion Y, and Apter A. 2011. Aggression, impulsivity, and suicide behavior: a review of the literature. Arch Suicide Res 15:93-112.

Hjelmeland H, Dieserud G, Dyregrov K, Knizek BL, and Leenaars AA. 2012. Psychological autopsy studies as diagnostic tools: are they methodologically flawed? Death Stud 36:605-626.

Klonsky ED, Alexis M. May. 2015. Impulsivity and suicide risk: review and clinical implications. vol. 32, no. 8, p. 13. Gale Academic OneFile. Accessed 14 Mar. 2021. Psychiatric Times.

Li M, and Katikireddi SV. 2019. Urban-rural inequalities in suicide among elderly people in China: a systematic review and meta-analysis. Int J Equity Health 18:2.

Li X, Xiao Z, and Xiao S. 2009. Suicide among the elderly in mainland China. Psychogeriatrics 9:62-66.

Liu BP, Qin P, and Jia CX. 2018. Behavior Characteristics and Risk Factors for Suicide Among the Elderly in Rural China. J Nerv Ment Dis 206:195-201.

Lu CF, Jia CX, Xu AQ, Dai AY, and Qin P. 2012. Psychometric characteristics of Chinese version of Barratt Impulsiveness Scale-11 in suicides and living controls of rural China. Omega (Westport) 66:215-229.

Ma Z, He Q, Nie G, Jia C, and Zhou L. 2020. Reliability and validity of short Beck Hopelessness Scale in psychological autopsy study among Chinese rural elderly. Int Psychogeriatr 32:525-531.

Mann JJ, Waternaux C, ., Haas GL, and Malone KM. 1999. Toward a clinical model of suicidal behavior in psychiatric patients. American Journal of Psychiatry 156:181.

McHugh CM, Chun Lee RS, Hermens DF, Corderoy A, Large M, and Hickie IB. 2019. Impulsivity in the self-harm and suicidal behavior of young people: A systematic review and meta-analysis. J Psychiatr Res 116:51-60.

Milner A, Sveticic J, and De Leo D. 2013. Suicide in the absence of mental disorder? A review of psychological autopsy studies across countries. Int J Soc Psychiatry 59:545-554.

Mo Q, Zhou L, He Q, Jia C, and Ma Z. 2019. Validating the Life Events Scale for the Elderly with proxy-based data: A case-control psychological autopsy study in rural China. Geriatr Gerontol Int 19:547-551.

Moeller FG, Barratt ES, Dougherty DM, Schmitz JM, and Swann AC. 2001. Psychiatric aspects of impulsivity. American Journal of Psychiatry 158:1783-1793.

Neufeld E, and O'Rourke N. 2009. Impulsivity and hopelessness as predictors of suicide-related ideation among older adults. Can J Psychiatry 54:684-692.

Pan YF, Ma ZY, Zhou L, and Jia CX. 2020. Psychometric Characteristics of Duke Social Support Index Among Elderly Suicide in Rural China. Omega (Westport) 82:105-119.

Paris J. 2005. The development of impulsivity and suicidality in borderline personality disorder. Dev Psychopathol 17:1091-1104.

Patton JH, Stanford MS, and Barratt ES. 1995. Factor structure of the Barratt impulsiveness scale. J Clin Psychol 
393

394

395

396

397

398

399

400

401

402

403

404

405

406

407

408

409

410

411

412

413

414

415

416
51:768-774.

Pouliot L, and De Leo D. 2006. Critical issues in psychological autopsy studies. Suicide Life Threat Behav 36:491-510. Sha F, Yip PSF, and Law YW. 2017. Decomposing change in China's suicide rate, 1990-2010: ageing and urbanisation. Injury Prevention Journal of the International Society for Child \& Adolescent Injury Prevention 23:injuryprev-2016-042006.

Smith AR, Witte TK, Teale NE, King SL, Bender TW, and Joiner TE. 2008. Revisiting impulsivity in suicide: implications for civil liability of third parties. Behav Sci Law 26:779-797.

Swahn MH, Ali B, Bossarte RM, Van Dulmen M, Crosby A, Jones AC, and Schinka KC. 2012. Self-harm and suicide attempts among high-risk, urban youth in the U.S.: shared and unique risk and protective factors. Int J Environ Res Public Health 9:178-191.

Turecki G, Brent DA, Gunnell D, O'Connor RC, Oquendo MA, Pirkis J, and Stanley BH. 2019. Suicide and suicide risk. Nat Rev Dis Primers 5:74.

Wang CW, Chan CL, and Yip PS. 2014. Suicide rates in China from 2002 to 2011: an update. Soc Psychiatry Psychiatr Epidemiol 49:929-941.

WHO WHO. 2019. World Health Organization. (2019). Mental health. Retrieved from https://www.who.int/gho/mental_health/en/.

Zhang J, and Jia CX. 2007. Validating a short version of the Suicide Intent Scale in China. Omega (Westport) 55:255265.

Zhang J, Li N, Tu XM, Xiao S, and Jia C. 2011. Risk factors for rural young suicide in China: a case-control study. J Affect Disord 129:244-251.

Zhang J, and Li Z. 2013. Characteristics of Chinese rural young suicides by pesticides. Int J Soc Psychiatry 59:655-662.

Zhou L, Wang GJ, Jia CX, and Ma ZY. 2019. Being left-behind, mental disorder, and elderly suicide in rural China: a case-control psychological autopsy study. Psychol Med 49:458-464. 


\section{Table $\mathbf{1}$ (on next page)}

Summary information of Suicides and living controls 
1 Table 1. Summary information of Suicides and living controls

\begin{tabular}{|c|c|c|c|c|}
\hline Variables & Suicides & Living controls & $\chi^{2} / \mathrm{F} / \mathrm{z}$ & $\mathrm{p}$ \\
\hline $\mathbf{N}$ & 242 & 242 & - & - \\
\hline Gender & & & - & - \\
\hline Male & $135(55.8)$ & $135(55.8)$ & & \\
\hline Female & $107(44.2)$ & $107(44.2)$ & & \\
\hline Age (years) & & & 1.12 & 0.569 \\
\hline $60-69$ & $73(30.2)$ & $70(28.9)$ & & \\
\hline $70-79$ & $100(41.3)$ & $111(45.9)$ & & \\
\hline$\geq 80$ & $69(28.5)$ & $61(25.2)$ & & \\
\hline Education & & & 1.92 & 0.383 \\
\hline Below primary school & $111(45.9)$ & $96(39.7)$ & & \\
\hline Primary school & $105(43.4)$ & $116(47.9)$ & & \\
\hline Above primary school & $26(10.7)$ & $30(12.4)$ & & \\
\hline Marital status & & & 19.89 & $<0.001$ \\
\hline Currently married a & $122(50.4)$ & $170(70.2)$ & & \\
\hline Not currently married ${ }^{b}$ & $120(49.6)$ & $72(29.8)$ & & \\
\hline Employment & & & 7.84 & 0.020 \\
\hline Employed & $40(16.5)$ & $59(24.4)$ & & \\
\hline Unemployed & $195(80.6)$ & $169(69.8)$ & & \\
\hline Retired & $7(2.9)$ & $14(5.8)$ & & \\
\hline \multicolumn{5}{|l|}{ Family annual income } \\
\hline$\left(\mathrm{CNY}^{\mathrm{c}}\right)$ & & & 1.87 & 0.394 \\
\hline$<3600$ & $88(36.4)$ & $74(30.6)$ & & \\
\hline $3600-9999$ & $88(36.4)$ & $98(40.5)$ & & \\
\hline$\geq 10000$ & $66(27.3)$ & $70(28.9)$ & & \\
\hline
\end{tabular}




\begin{tabular}{|c|c|c|c|c|}
\hline Being left-behind & & & 4.49 & 0.034 \\
\hline Yes & $41(16.9)$ & $25(10.3)$ & & \\
\hline No & $201(83.1)$ & $217(89.7)$ & & \\
\hline Physical diseases & & & 18.52 & $<0.001$ \\
\hline Yes & $202(83.5)$ & $161(66.5)$ & & \\
\hline No & $40(16.5)$ & $81(33.5)$ & & \\
\hline Total scores of negative life events & $49.76 \pm 30.19$ & $25.24 \pm 28.46$ & 79.95 & $<0.001$ \\
\hline Social support ( mean \pm SD ) & $22.88 \pm 5.98$ & $27.47 \pm 6.81$ & 78.20 & $<0.001$ \\
\hline BIS score d & $98.79 \pm 16.63$ & $86.91 \pm 15.18$ & 78.99 & $<\mathbf{0 . 0 0 1}$ \\
\hline Nonplanning impulsivity & $35.18 \pm 7.24$ & $31.07 \pm 7.07$ & 45.34 & $<0.001$ \\
\hline Motor impulsivity & $28.43 \pm 8.28$ & $23.70 \pm 6.05$ & 50.91 & $<0.001$ \\
\hline Attentional impulsivity & $35.18 \pm 5.95$ & $32.13 \pm 5.67$ & 41.35 & $<0.001$ \\
\hline
\end{tabular}

2 a included married and living with spouse or cohabiting.

$3 \mathrm{~b}$ included single, divorced, widowed and married but living apart.

$4 \quad{ }^{\mathrm{c}} \mathrm{CNY}$, Chinese Yuan.

$5 \quad$ d BIS: Barratt Impulsiveness Scale.

6 
Table 2 (on next page)

Table2. Comparisons of suicide behaviors by high and low impulsivity group 
1 Table 2. Comparisons of suicide behaviors by high and low impulsivity group

\begin{tabular}{|c|c|c|c|c|}
\hline Variables & High impulsivity a & Low impulsivity & $\chi^{2 / t / z}$ & $\mathrm{p}$ \\
\hline Suicides & $120(49.6)$ & $122(50.4)$ & - & - \\
\hline Suicide time & & & 0.71 & 0.447 \\
\hline $6: 00-17: 59$ & $95(79.2)$ & $91(74.6)$ & & \\
\hline $18: 00-5: 59$ & $25(20.8)$ & $31(25.4)$ & & \\
\hline Suicide place & & & 0.19 & 0.700 \\
\hline Home & $104(86.7)$ & $108(88.5)$ & & \\
\hline Others & $16(13.3)$ & $14(11.5)$ & & \\
\hline Suicide means & & & 1.199 & 0.945 \\
\hline Pesticides & $63(52.5)$ & $62(50.8)$ & & \\
\hline Hanging & $45(37.5)$ & $50(41.0)$ & & \\
\hline Drowning & $5(4.2)$ & $4(3.3)$ & & \\
\hline Non-pesticides poisoning & $5(4.2)$ & $3(2.5)$ & & \\
\hline Jumping off a building & $1(0.8)$ & $2(1.6)$ & & \\
\hline Cutting throat & $1(0.8)$ & $1(0.8)$ & & \\
\hline History of attempted suicide & & & 8.24 & 0.005 \\
\hline Yes & $31(25.8)$ & $14(11.5)$ & & \\
\hline No & $89(74.2)$ & $108(88.5)$ & & \\
\hline Suicide intent & $4.93 \pm 2.58$ & $4.85 \pm 2.41$ & -0.23 & 0.821 \\
\hline
\end{tabular}

$2{ }^{a}$ high impulsivity was defined by BIS score above the median. 
Table 3 (on next page)

Risk factors of suicide among rural elderly in China 
1 Table 3. Risk factors of suicide among rural elderly in China

\begin{tabular}{|c|c|c|}
\hline Independent variables & OR $(95 \% \mathrm{CI})$ & $\mathrm{p}$ \\
\hline Impulsivity & $1.03(1.01-1.06)$ & 0.015 \\
\hline \multicolumn{3}{|l|}{ Marital status } \\
\hline Currently married & $1(\mathrm{ref})$ & \\
\hline Not Currently married & $2.26(1.04-4.92)$ & 0.039 \\
\hline \multicolumn{3}{|l|}{ Being left-behind } \\
\hline No & 1 (ref) & \\
\hline Yes & $0.63(0.22-1.83)$ & 0.392 \\
\hline Social support & $0.93(0.88-0.99)$ & 0.023 \\
\hline \multicolumn{3}{|l|}{ Education } \\
\hline Below primary school & 1 (ref) & \\
\hline Primary school & $1.58(0.43-5.86)$ & 0.491 \\
\hline Above primary school & $0.72(0.24-2.11)$ & 0.547 \\
\hline \multicolumn{3}{|l|}{ Family annual income } \\
\hline \multicolumn{3}{|l|}{$\left(\mathrm{CNY}^{\mathrm{a}}\right)$} \\
\hline$<3600$ & 1 (ref) & \\
\hline $3600-9999$ & $0.59(0.24-1.43)$ & 0.241 \\
\hline$\geq 10000$ & $0.51(0.21-1.25)$ & 0.143 \\
\hline Total scores of negative life events & $1.02(1.01-1.03)$ & 0.001 \\
\hline
\end{tabular}

$2{ }^{a} \mathrm{CNY}$, Chinese Yuan. 